Pacific Journal of Mathematics

THREE REMARKS ON SYMMETRIC PRODUCTS AND 


\section{THREE REMARKS ON SYMMETRIC PRODUCTS AND SYMMETRIC MAPS}

\section{V.P. SNAITH AND J. J. UCCI}

The first remark establishes that the homotopy type of a certain space related to the $m$-fold symmetric product $S P^{m} S^{n}$ of the $n$-sphere is that of an $n^{\text {th }}$ suspension space. Remark two generalizes a well-known adjunction formula for $S P^{2} S^{n}$ due to Steenrod to a filtration of length $m$ of $S P^{m} S^{n}$. The final remark provides a group-theoretic construction of $G$ maps $f:\left(S^{n}\right)^{m} \rightarrow S^{n}$ where $G \subset S(m)$ acts on $\left(S^{n}\right)^{m}$ by permutation of its factors.

1. Joins. The join $X * Y$ of $X$ and $Y$ is the quotient space $X \times Y \times I / \sim$ where $(x, y, 0) \sim\left(x, y^{\prime} ; 0\right)$ and $(x, y, 1) \sim\left(x^{\prime}, y, 1\right)$ for all $x, x^{\prime} \in X$ and all $y, y^{\prime} \in Y$. Let $(D, S)$ denote the unit disc and sphere in euclidean $n$-space $R^{n}$ with its usual inner product

$$
\langle x, y\rangle=\Sigma x_{i} y_{i}
$$

For any decomposition $R^{n}=W_{1} \oplus W_{2}$ of $R^{n}$ into the direct sum of a $k$-dimensional subspace $W_{1}$ and its orthogonal complement $W_{2}=W_{1}^{\perp}$, let $D_{i}=D \cap W_{i}$ and $S_{i}=S \cap W_{i}, i=1,2$, be the associated discs and spheres. As well known the map $f: D_{1} * S_{2} \rightarrow D$ given by

$$
f[s x, y, t]=s \sqrt{1-t} x+\sqrt{t} y
$$

defines a homeomorphism of pairs

$$
\left(D_{1} * S_{2}, S_{1} * S_{2}\right) \cong(D, S) \text {. }
$$

Give $V_{i}=R^{n}, i=1, \cdots, n$, its usual inner product $\langle,\rangle_{i}$. Then the formula $\langle x, y\rangle=\Sigma\left\langle x_{i}, y_{i}\right\rangle_{i}$ for $x=\left(x_{1}, \cdots, x_{n}\right), y=\left(y_{1}, \cdots, y_{n}\right)$ in $V=V_{1} \times \cdots \times V_{m} \cong R^{n m}$ coincides with the usual inner product on $R^{n m}$. So we may apply the preceding remarks to the diagonal subspace $W_{1}=\left\{v \in V \mid v_{1}=v_{2}=\cdots=v_{m}\right\}$ and its orthogonal complement $W_{2}=\left\{v \in V \mid \Sigma v_{i}=0\right\}=W_{1}^{\perp}$. The full symmetric group $S(m)$ acts on $V$ by permutation of its factors $V_{i}$. For any subgroup $H$ of $S(m)$ $D_{i}$ and $S_{i}$ are $H$-spaces and $f$ an $H$-map inducing another homeomorphism of pairs

$$
\left(D_{1} / H * S_{2} / H, S_{1} / H * S_{2} / H\right) \cong(D / H, S / H)
$$

As $H$ acts trivially on $W_{1}, D_{1} / H=\bar{D}_{1}$ and $S_{1} / H=\bar{S}_{1}$ are again the disc and sphere. Moreover, for subgroups $H_{1} \subset H_{2}$ of $S(m)$ it is easily checked that the quotient map $p: D / H_{1} \rightarrow D / H_{2}$ corresponds via 
(2) to the join map

$$
i d * p_{2}: \bar{D}_{1} *\left(S_{2} / H_{1}\right) \longrightarrow \bar{D}_{1} *\left(S_{2} / H_{2}\right) \text {. }
$$

Recall from [7] the definition of the spaces $X_{m, l}^{n}$ which appear in the geometry of the symmetric product $S P^{m} S^{n}$. Let $h_{\tau}:\left(D^{n}\right)^{m} \rightarrow\left(D^{n}\right)^{m}$ be the permutation homeomorphism defined by $\tau \in S(m)$, and set

$$
A_{m, l}^{n}=\left(D^{n}\right)^{m-l} \times\left(S^{n-1}\right)^{l} \quad \text { for } 0 \leqslant l \leqslant m .
$$

Then

$$
\widetilde{X}_{m, l}^{n}=\bigcup_{\tau \in S(m)} h_{\tau}\left(A_{m, l}^{n}\right)
$$

is an $S(m)$-subspace of $\left(D^{n}\right)^{m}$ and so $X_{m, l}^{n}=\widetilde{X}_{m, l}^{n} / S(m)$ is well defined. To identify the pairs $(D / S(m), S / S(m))$ and $\left(X_{m, 0}^{n}, X_{m, 1}^{n}\right)$ we make the following change of norms: let $V^{\prime}=V$ as sets but set

$$
\|v\|^{\prime}=\max _{i}\left\|v_{i}\right\|_{i}
$$

where $\left\|v_{i}\right\|_{i}=\left\langle v_{i} \cdot v_{i}\right\rangle_{i}^{1 / 2}$. Then $x \rightarrow\left(\|x\| /\|x\|^{\prime}\right) \cdot x$ defines a norm preserving (non-linear) $S(m)$-homeomorphism $V \rightarrow V^{\prime}$ establishing the desired result $(D / S(m), S / S(m)) \cong\left(X_{m, 0}^{n}, X_{m, 1}^{n}\right)$. Thus

$$
\left(\bar{D}_{1} *\left(S_{2} / S(m)\right), \bar{S}_{1} *\left(S_{2} / S(m)\right)\right) \text { and }\left(X_{m, 0}^{n}, X_{m, 1}^{n}\right)
$$

are homeomorphic pairs. Moreover the canonical map $D^{n} \times X_{m-1,0}^{n} \rightarrow$ $X_{m, 0}^{n}$ is the quotient map $\left(D^{n}\right)^{m} / S(m-1) \rightarrow\left(D^{n}\right)^{m} / S(m)$ induced by the inclusion homomorphism $S(m-1) \rightarrow S(m)$ sending $S(m-1)$ onto the subgroup $\{e\} \times S(m-1)$ of $S(m)$ which acts on $\left(D^{n}\right)^{m}=D^{n} \times\left(D^{n}\right)^{m-1}$ by the identity on the first factor and by the usual symmetric action on the second factor. Combining the remark of the preceding paragraph with the above $S(m)$-homeomorphism $V \rightarrow V^{\prime}$ we see that the canonical map $D^{n} \times X_{m-1,0}^{n} \rightarrow X_{m, 0}^{n}$ corresponds to the join map

$$
\bar{D}_{1} *\left(S_{2} / S(m-1)\right) \longrightarrow \bar{D}_{1} *\left(S_{2} / S(m)\right) \text {. }
$$

Our first remark establishes a conjecture stated in [7].

Proposition 1.1. $X_{m, m-1}^{n} / X_{m-1, m-2}^{n}$ has the homotopy type of a space of the form $S^{n-1} * K$ for $K$ a suitable finite $C W$ complex. Hence $X_{m, m-1}^{n} / X_{m-1, m-2}^{n}$ has the homotopy type of an $n^{\text {th }}$ suspension.

Proof. Proposition 2.6 of [7] asserts the existence of a homotopy equivalence

$$
X_{m, m-1}^{n} / X_{m-1, m-2}^{n} \sim E X_{m, 1}^{n-1} \bigcup_{E \psi} C\left(E\left(X_{1,1}^{n-1} * X_{m-1,1}^{n-1}\right)\right)
$$


where $\psi$ is given by the canonical maps

$$
X_{1,1}^{n-1} \times X_{m-1,0}^{n-1} \longrightarrow X_{m, 1}^{n-1}, \quad X_{1,0}^{n-1} \times X_{m-1,1}^{n-1} \longrightarrow X_{m, 1}^{n-1} .
$$

$\psi$ is just the restriction of the canonical map

$$
\psi^{\prime}: X_{1,0}^{n-1} \times X_{m-1,0}^{n-1} \longrightarrow X_{m, 0}^{n-1}
$$

which, as we have already noted, can be identified with the join map $i d * \bar{\psi}^{\prime}: \bar{D}_{1} *\left(S_{2} / S(m-1)\right) \longrightarrow \bar{D}_{1} *\left(S_{2} / S(m)\right)$. Under this identification the subspaces $X_{1,1}^{n-1} \times X_{m-1,0}^{n-1} \cup X_{1,0}^{n-1} \times X_{m-1,1}^{n-1}$ and $X_{m, 1}^{n-1}$ correspond to $\bar{S}_{1} *\left(S_{2} / S(m-1)\right)$ and $\bar{S}_{1} *\left(S_{2} / S(m)\right)$, and so the map $\psi$ corresponds to the join map $i d * \bar{\psi}: \bar{S}_{1} *\left(S_{2} / S(m-1)\right) \rightarrow \bar{S}_{1} *\left(S_{2} / S(m)\right)$ which is the restriction of $i d * \bar{\psi}^{\prime}$. Hence there is a homotopy equivalence

$$
\begin{aligned}
X_{m, m-1}^{n} / X_{i n-1, m-2}^{n} & \sim E\left(\left(E^{n-1}\left(S_{2} / S(m)\right)\right) \underset{E^{n-1} \bar{\psi}}{\bigcup} C\left(E^{n-1} X_{m-1,1}^{n-1}\right)\right) \\
& \sim E^{n}\left(\left(S_{2} / S(m)\right) \bigcup_{\bar{\psi}} C X_{m-1,1}^{n-1}\right.
\end{aligned}
$$

and the result is proved.

For $p$-fold cyclic products ( $p$ any prime) there is an analogous result to 1.1 whose proof differs only slightly from the preceding. For this let now $h_{:}:\left(D^{n}\right)^{p} \rightarrow\left(D^{n}\right)^{p}$ be the (cyclic) permutation homeomorphism defined by $\tau \in Z_{p} \subset S(p)$ and set $A_{p, l}^{n}=\left(D^{n}\right)^{p-l} \times\left(S^{n-1}\right)^{l}$. Then as before

$$
\widetilde{X}_{p, l}^{n}=\bigcup_{\tau \in Z_{p}} h_{\tau}\left(A_{p, l}^{n}\right)
$$

is a $Z_{p}$-space and $X_{p, l}^{n}=\widetilde{X}_{p, l}^{n} / Z_{p}$ is well defined. Let $W_{l}^{n-1} \subset\left(S^{n-1}\right)^{l}$ be the subspace $\left\{x \in\left(S^{n-1}\right)^{l} \mid x_{i}=\right.$ basepoint for some $\left.i\right\}, \widetilde{Z}_{p, l}^{n}=\left(D^{n}\right)^{p-l} \times$ $W_{l}^{n-1}$ and $Z_{p, l}^{n}$ the image of $\widetilde{Z}_{p, l}^{n}$ under the canonical projection

$$
\left(D^{n}\right)^{p-l} \times\left(S^{n-1}\right)^{l} \longrightarrow X_{p, l}^{n} \text {. }
$$

Then $Z_{p, p-1}^{n} \subset X_{p, p-1}^{n}$ and formula (3.3) of [8] asserts the existence of a homeomorphism

$$
X_{p, p-1}^{n} / Z_{p, p-1}^{n} \cong E X_{p, 1}^{n-1} \cup e^{n p-p+1} .
$$

The top cell $e^{n p-p+1}$ arises from the product $D^{n} \times\left(D^{n-1}\right)^{p-1}$ and the attaching map of (4) $S^{n-1} \times\left(D^{n-1}\right)^{p-1} \cup D^{n} \times \partial\left[\left(D^{n-1}\right)^{p-1}\right] \rightarrow E X_{p, 1}^{n-1}$ sends the contractible subspace

$$
A=S^{n-1} \times \partial\left[\left(D^{n-1}\right)^{p-1}\right] \cup \text { point } \times\left(D^{n-1}\right)^{p-1}
$$

to the basepoint of $E X_{p, 1}^{n-1}$ and so factors as $(E \psi) \circ p$, where $p$ is the collapsing homotopy equivalence 


$$
S^{n-1} * \partial\left[\left(D^{n-1}\right)^{p-1}\right] \longrightarrow S^{n-1} * \partial\left[\left(D^{n-1}\right)^{p-1}\right] / A
$$

and $\psi$ the canonical projections

$$
S^{n-2} \times\left(D^{n-1}\right)^{p-1} \longrightarrow X_{p, 1}^{n-1}, \quad D^{n-1} \times \partial\left[\left(D^{n-1}\right)^{p-1}\right] \longrightarrow X_{p, 1}^{n-1}
$$

(see the proof of Prop. 2.6 of [7] with $Z_{p}$ replacing $S(m)$ ). By the above $X_{p, 1}^{n-1}$ is homeomorphic to the join $S_{1} *\left(S_{2} / Z_{p}\right)-V$ is now $R^{n p}$ and the map $\psi$ can be identified via this homeomorphism with the join map $i d * p: S_{1} * S_{2} \rightarrow S_{1} *\left(S_{2} / Z_{p}\right)$. Thus we obtain the analogous result to 1.1 .

Proposition 1.2. For the p-fold cyclic product of spheres the space $X_{p, p-1}^{n} / Z_{p, p-1}^{n}$ has the homotopy type of a space of the form $S^{n-1} * K$ for $K$ a suitable finite $C W$ complex. Hence $X_{p, p-1}^{n} / Z_{p, p-1}^{n}$ has the homotopy type of an $n^{\text {th }}$ suspension.

Application of 1.2 was made in [8].

Consider again the symmetric product situation. Lemma 2.5 (iii) of [7] provides a homeomorphism

$$
X_{m, l}^{n} / X_{m-1, l-1}^{n} \cong\left(X_{m, l+1}^{n} / X_{m-1, l}^{n}\right) \cup C\left(X_{m-l, 1}^{n} * X_{l, 1}^{n-1}\right) .
$$

For $l=m$ and $l=m-1$ the spaces $X_{m, l}^{n} / X_{m-1, l-1}^{n}$ have now been shown to have the homotopy type of a space of the form $S^{n-1} * K$. It seems reasonable to expect the same to be true for the remaining values of $l, 2 \leqslant l \leqslant m-2$.

2. Geometry of $S P^{m} E X$. Our second remark extends the Steenrod adjunction formula [3]

$$
S P^{2} S^{n} \cong E\left(S P^{2} S^{n-1}\right) \cup e^{2 n}
$$

to higher symmetric products $S P^{m} E X$ of suspension spaces. Let

$$
\begin{aligned}
I^{n} & =\left\{x \in R^{n} \mid 0 \leqslant x_{i} \leqslant 1\right\} \\
A_{n} & =\left\{x \in I^{n} \mid x_{n}=1\right\} \\
T_{n} & =\left\{x \in I^{n} \mid x_{1} \geqslant x_{2} \geqslant \cdots \geqslant x_{n}\right\} \\
p & =\{(1,1, \cdots, 1)\} \subset I^{n} .
\end{aligned}
$$

For $i=1,2, \cdots, n-1$ define $f_{i}: I^{n} \rightarrow I^{n}$ by $f_{i}\left(t_{1}, \cdots, t_{n}\right)=\left(t_{1}^{\prime}, \cdots, t_{n}^{\prime}\right)$ where $t_{j}^{\prime}=t_{j}$ if $j \neq i$ and $t_{i}^{\prime}=t_{i+1}+t_{i}\left(1-t_{i+1}\right)$. One shows easily that the composite $g_{n}=f_{1} \circ f_{2} \circ \cdots \circ f_{n-1}$ defines a relative homeomorphism $\left(I^{n}, A_{n}\right) \cong\left(T_{n}, p\right)$. The map $g_{n}$ is useful in studying the quotients $A_{i+1} / A_{i}$ arising from a filtration 


$$
S P^{m} E X=A_{m} \supset A_{m-1} \supset \cdots \supset A_{1}=E\left(S P^{m} X\right)
$$

which we define as follows. For $x^{\prime}=[x, t] \in E X$ call $t$ the height of $x^{\prime}$. As each element $\left[x_{1}^{\prime}, \cdots, x_{m}^{\prime}\right] \in S P^{m} E X$ has a representative with heights $t_{1} \geqslant t_{2} \geqslant \cdots \geqslant t_{m}$ we can set $A_{i}$ to be the subset of $S P^{m} E X$ of all elements having representatives with at most $i$ distinct heights. The $A_{i}$ define a filtration (5) of $S P^{m} E X$.

For any partition $\pi=\left[i_{1}: i_{2}: \cdots: i_{q}\right]$ of $m$ let $A_{q \pi} \subset A_{q}$ be the set of all points having representatives with heights $t_{1} \geqslant t_{2} \geqslant \cdots \geqslant t_{q}, i_{1}$ of the $m$ coordinates at height $t_{1}, i_{2}$ of them at height $t_{2}$, etc. Set $Y_{1}=C\left(S P^{i_{1}} X\right), Y_{j}=\left(S P^{i_{j}} X\right) \times I$ for $2 \leqslant j<q, Y_{q}=\widetilde{C}\left(S P^{i_{q}} X\right)$ and $Y=Y_{1} \times \cdots \times Y_{q}$ where

$$
C(Z)=Z \times I / Z \times\{1\} \quad \text { and } \quad \widetilde{C}(Z)=Z \times I / Z \times\{0\} .
$$

Set

$$
\partial C(Z)=\{[z, t] \in C(Z) \mid t=0\}, \partial \widetilde{C}(Z)=\{[z, t] \in \widetilde{C}(Z) \mid t=1\}
$$

and

$$
\partial\left(S P^{k} X \times I\right)=S P^{k} X \times\{0,1\} .
$$

This defines $\partial Y_{i}$ for $i=1, \cdots, q$. Finally set

$$
\partial Y=\bigcup_{i=1}^{q} Y_{1} \times \cdots \times \partial Y_{i} \times \cdots \times Y_{q} .
$$

Clearly $\partial$ is just a kind of boundary operator for cones and related spaces.

Proposition 2.1. The map $Y \rightarrow S P^{m} E X$ given by

$$
\begin{aligned}
& \left(\left[x_{1}, t_{1}\right],\left(x_{2}, t_{2}\right), \cdots,\left(x_{q-1}, t_{q-1}\right),\left[x_{q}, t_{q}\right]\right) \\
\longrightarrow & {\left[\left[x_{1}, t_{1}^{\prime \prime}\right],\left[x_{2}, t_{2}^{\prime \prime}\right], \cdots,\left[x_{q}, t_{q}^{\prime \prime}\right]\right] }
\end{aligned}
$$

where $t_{j}^{\prime \prime}$ is the $j^{\text {th }}$ coordinate of $g_{q}\left(t_{1}, \cdots, t_{q}\right)$, induces a relative homeomorphism $(Y, \partial Y) \cong\left(A_{q \pi}, A_{q-1}\right)$ for each $2 \leqslant q<m$ and each partition $\pi=\left[i_{1}: \cdots: i_{q}\right]$ of $m$.

The proof is straighforward.

To obtain an expression for $A_{q} / A_{q-1}$ first observe that

$$
A_{q} / A_{q-1}=\bigvee_{\pi}\left(A_{q \pi} / A_{q-1}\right),
$$

the wedge taken over all partitions of $m$. Thus by 2.1 it suffices to consider for each $\pi$ the corresponding quotient $Y / \partial Y$. 
Proposition 2.2. For $\pi=\left[i_{1}: \cdots: i_{q}\right]$ and $Y=Y_{1} \times \cdots \times Y_{q}$ as above the space $Y / \partial Y$ has the same homotopy type as the space

$$
E^{q}\left(S P^{i_{1}} X \wedge S P^{i_{q}} X\right) \vee E^{q}\left(S P^{i_{1}} X \wedge \prod_{j=2}^{q-1} S P^{i_{j}} X \wedge S P^{i_{q}} X\right)
$$

Proof. Let $B$ be a space with $\partial B=\phi$. Then the obvious quotient map $Q=C A \times B \times I^{q-2} \times C A^{\prime} \rightarrow P=E A \times B \times S^{q-2} \times E A^{\prime}$ induces a relative homeomorphism $(Q, \partial Q)=\left(P, P^{\prime}\right)$ where

$$
P^{\prime}=E A \times B \times S^{q-2} \vee E A \times B \times E A^{\prime} \vee B \times S^{q-2} \times E A^{\prime} .
$$

So $P / P^{\prime}=B \times\left(E A \wedge S^{q-2} \wedge E A^{\prime}\right) / B \times$ point and the latter quotient has the homotopy type of the wedge $E^{q}\left(A \wedge A^{\prime}\right) \bigvee E^{q}\left(A \wedge B \wedge A^{\prime}\right)$ [5]. Therefore 2.2 is obtained by setting $A=S P^{i_{1}} X, B=\prod_{j=2}^{q-1} S P^{i_{j}} X$ and $A^{\prime}=S P^{i_{q}} X$.

As an illustration of the preceding analysis let us return to the Steenrod formula for the symmetric square of a sphere. The space $Y$ is just $C X \times \widetilde{C} X$ and the map $C X \times \widetilde{C} X \rightarrow S P^{2} E X$ is

$$
\left(\left[x_{1}, t_{1}\right],\left[x_{2}, t_{2}\right]\right) \longmapsto\left[\left[x_{1}, t_{2}+t_{1}\left(1-t_{2}\right)\right],\left[x_{2}, t_{2}\right]\right] .
$$

The subspace $X \times \widetilde{C} X \cup C X \times X$ (given by $t_{1}=0$ or $t_{2}=1$ ) is mapped to $E S P^{2} X$. It is well known that there are homeomorphisms

$$
X \times \widetilde{C} X \cup C X \times X \cong X * X
$$

and

$$
C X \times \widetilde{C} X \cong C(X \times \widetilde{C} X \cup C X \times X) .
$$

Hence we obtain the adjunction formula $S P^{2} E X \cong E S P^{2} X \cup C(X * X)$ extending the Steenrod result from spheres to suspensions.

REMARK. For $X=S^{n-1} 2.2$ can be used to recompute Nakaoka's results [4] on the integral cohomology of $S P^{m} S^{n}$ for low values of $m$.

3. Group theoretic construction of symmetric maps. Let $H \subset G \subset S(m)$ be subgroups of the symmetric group $S(m)$ and let $S(G / H)$ be the symmetric group on the set of right cosets $G / H$. Define a homomorphism $\alpha: G \rightarrow S(G / H)$ by $\alpha(g)\left(H g_{1}\right)=H g_{1} g^{-1}$. Kernel of $\alpha$ is just the normal subgroup $B=\bigcap_{g \in G} g H^{-1}$ and so there is an injection $G / B \rightarrow S(G / H)$. Let $A$ denote the image of $\alpha$ and $|G / H|$ the cardinality of $G / H$.

Proposition 3.1. If $v: X^{|G| H \mid} \rightarrow X$ and $w: X^{m} \rightarrow X$ are $A$ and $H$-maps respectively, then $F: X^{m} \rightarrow X$ given by 


$$
F(x)=v\left(w\left(g_{1} \cdot x\right), w\left(g_{2} \cdot x\right), \cdots, w\left(g_{l} \cdot x\right)\right)
$$

for $g_{1}, \cdots, g_{l}$ a complete set of coset representatives in $G / H$, is a $G-\operatorname{map}$.

Proof. As $w$ is an $H$-map we have for any $g \in G$ and any

$$
1 \leqslant i \leqslant l=|G / H|
$$

the existence of an $h \in H$ and a unique $1 \leqslant j \leqslant l$ such that

$$
w\left(g_{i} \cdot(g \cdot x)\right)=w\left(h \cdot\left(g_{j} \cdot x\right)\right)=w\left(g_{j} \cdot x\right),
$$

where $h$ arises from the coset equality $H g_{i} g=H g_{j}$. Hence there exists an element $\sigma \in S(G / H)$ in $A=$ image $(\alpha)$ satisfying

$$
\begin{aligned}
F(g \cdot x) & =v\left(w\left(g_{1} \cdot(g \cdot x)\right), \cdots, w\left(g_{l} \cdot(g \cdot x)\right)\right) \\
& =v\left(w\left(g_{\sigma(1)} \cdot x\right), \cdots, w\left(g_{\sigma(l)} \cdot x\right)\right) \\
& =v\left(\sigma \cdot\left(w\left(g_{1} \cdot x\right), \cdots, w\left(g_{l} \cdot x\right)\right)\right) \\
& =v\left(w\left(g_{1} \cdot x\right), \cdots, w\left(g_{l} \cdot x\right)\right)=F(x) .
\end{aligned}
$$

The result follows.

To compute the James number of $F$ when $X=S^{n}$ note that the degree of the composite $S^{n} \stackrel{\Delta}{\longrightarrow}\left(S^{n}\right)^{m} \stackrel{F}{\longrightarrow} S^{n}$ ( $\Delta$ the diagonal map) equals the product $\operatorname{deg}(v \circ \Delta) \cdot \operatorname{deg}(w \circ \Delta)$, since $F \circ \Delta=v \circ \Delta \circ w \circ \Delta$ as maps. Therefore the James number of $F$ is easily computed from those of $v$ and $w$ via the Künneth formula.

Applications. Let $n=2 t+1$ in the following four applications.

1. Let $H=\{i d,(123),(132)\} \cong Z_{3}$ so $H \triangleleft S(3)=G$. Choose $v:\left(S^{n}\right)^{|G| H \mid} \rightarrow S^{n}$ to be an $S(2)$-map with $J_{v}=2^{\dot{\phi}(2 t)}[2]$ and $w:\left(S^{n}\right)^{3} \rightarrow S^{n}$ to be an $H$-map with $J_{w}=3^{t}$ [8]. Then $J_{F}=2^{\dot{\phi}(2 t)+1} \cdot 3^{t}$. However obstruction theory can improve this result as follows. From [7] we know that there exists a map $S P^{m} S^{n} \rightarrow S^{n}$ of James number $N$ if and only if the composite $X_{m, m-1}^{n} \stackrel{\phi}{\longrightarrow} S^{n} \stackrel{f_{N}}{\longrightarrow} S^{n}$, is nullhomotopic where $\operatorname{deg} f_{N}=N$. Here $\phi$ arises from the geometry of $S P^{m} S^{n}$ given in [7, §2]. As $X_{2,1}^{n} \subset X_{3,2}^{n}$ the obstructions to extending an $S(2)$-map $g_{1}:\left(S^{n}\right)^{2} \rightarrow S^{n}$ to an $S(3)$-map $g:\left(S^{n}\right)^{3} \rightarrow S^{n}$ lie in the groups $H^{i}\left(X_{3,2}^{n}, X_{2,1}^{n} ; \pi_{i} S^{n}\right)$, which by Nakaoka [4] (see also [1], Lemma (4.3)) are 3-primary. Hence there exists an $S(3)$-map $G:\left(S^{n}\right)^{3} \rightarrow S^{n}$ with $J_{G}=2^{\dot{\delta}(2 t)} \cdot 3^{r}$ for some $r$. As the set of all possible James numbers of $S(m)$-maps forms an ideal [1], there must also exist an $S(3)$-map $G^{\prime}:\left(S^{n}\right)^{3} \rightarrow S^{n}$ with $J_{G^{\prime}}=2^{\dot{\phi}(2 t)} \cdot 3^{t}$ and so we recover the main result 
of [7].

2. Let $H \subset S(4)$ be the subgroup generated by $\{(12),(34),(13)(24)\}$, so $|H|=8, H \bowtie G$ and

$$
B=\bigcap_{g \in G} g H g^{-1}=\{i d,(14)(23),(13)(24),(12)(34)\} \cong Z_{2} \times Z_{2} .
$$

Hence $|B|=4$ and $A=S(3)$. Apply 3.1 with $v$ an $S(3)$-map with $J_{v}=2^{\phi(2 t)} \cdot 3^{t}$ and $w$ the $H$-map $\left(S^{n}\right)^{4} \stackrel{h \circ h^{2}}{\longrightarrow} S^{n}$ where $h:\left(S^{n}\right)^{2} \rightarrow S^{n}$ is an $S(2)$-map with $J_{h}=2^{\phi(2 t)}$. Clearly $J_{w}=2^{2 \cdot \phi(2 t)}$ and so we obtain an $S(4)$-map $F:\left(S^{n}\right)^{4} \rightarrow S^{n}$ with $J_{F}=2^{3 \phi(2 t)} \cdot 3^{t+1}$. Now an exactly analogous argument to that of (1) shows that the obstructions to extending an $S(3)$-map $\left(S^{n}\right)^{3} \rightarrow S^{n}$ to an $S(4)$-map $\left(S^{n}\right)^{4} \rightarrow S^{n}$ lie in the groups $H^{i}\left(X_{4,3}^{n}, X_{3,2}^{n} ; \pi_{i} S^{n}\right)$, which again by Nakaoka are 2-primary. Thus there is an $S(4)$-map of James number $J=2^{r} \cdot 2^{\phi(2 t)} \cdot 3^{t}$ for some $r$. This as above implies the existence of an $S(4)$-map with James number $2^{3 \phi(2 t)} \cdot 3^{t}$. Note it is not difficult using $K$-theory to show that the James number of any $S(4)$-map $\left(S^{n}\right)^{4} \rightarrow S^{n}$ must be a multiple of $2^{2 t} \cdot 3^{t}$ (the first named author has improved this bound to $2^{\phi(2 t)} \cdot 2^{t} \cdot 3^{t}$ via ad hoc considerations).

3. For $G=G^{r}$ the Sylow $p$-subgroup of $S\left(p^{r}\right)$ given by the $r$ fold Wreath product of $G^{1} \cong Z_{p}$ with itself and $H=\Pi_{k=1}^{p} G^{r-1} \triangleleft G=G^{r}$ (see $[8, \S 2]$ ) we have $G / H \cong Z_{p}$. Let $w$ be the composite

$$
\left(S^{n}\right)^{p^{r}} \stackrel{\pi_{1}}{\longrightarrow}\left(S^{n}\right)^{p^{r-1}} \stackrel{w_{1}}{\longrightarrow} S^{n}
$$

where $w_{1}$ is a $G^{r-1}$-map with James number $J_{w_{1}}$ and $\pi_{1}$ is projection onto the first $p^{r-1}$ factors of $\left(S^{n}\right)^{p^{r}}$; let $v:\left(S^{n}\right)^{p} \rightarrow S^{n}$ be a $Z_{p}$-map with James number $J_{v}$. Then $J_{F}=J_{w_{1}} \cdot J_{v}$ where $F$ is given by 3.1 . From a $Z_{p}$-map $h$ with $J_{h}=p^{t}$ [2], this result plus induction on $r$ provides a $G^{r}$-map $h^{\prime}$ with $J_{h^{\prime}}=p^{r t}$. This iteration of 3.1 applied to the $G^{1}$-map $h$ gives precisely the composite $G^{r}$-map $h \circ h^{p} \circ \ldots \circ h^{p^{r-1}}$ : $\left(S^{n}\right)^{p^{r}} \rightarrow S^{n}$.

4. For $G=Z_{m n}$ and $H=Z_{n} \triangleleft G$ we have $A=Z_{m}$. In this situation 3.1 provides a $G$-map $F$ with $J_{F}=J_{w} \cdot J_{v}$ where $w=w_{1} \circ \pi_{1}$ is the composite of a $Z_{n}$-map $w_{1}$ and projection $\pi_{1}: X^{m} \rightarrow X^{n}$. Thus 3.1 provides the construction of the "best" cyclic map of order $m$ from the "best" cyclic maps of prime-power orders occurring in the prime decomposition of $m$. The latter are studied in [6].

In conclusion we remark that if $B$ is the trivial subgroup, 3.1 provides no useful information at all e.g. $G=S(m)$ for $m \geqslant 5$. Also the appearance of obstruction theory in applications 1 and 2 above 
indicate the limitations of 3.1. It would appear now from the results of [9] that the most natural approach to constructing $S(m)$-maps of minimal James number is via obstruction theory using [8] and Nakaoka's results relating the cohomology of $S P^{m} S^{n}$ to that of iterated cyclic products of spheres.

\section{REFERENCES}

1. I. M. James, Symmetric function of several variables whose range and domain is a sphere, Bol. Soc. Mat. Maxicana, 1 (1956), 85-88.

2. I. M. James, E. Thomas, H. Toda and G. W. Whitehead, On the symmetric square of a sphere, J. Math. and Mech., 12 (1963), 771-776.

3. S. D. Liao, On the topology of cyclic products of spheres, Trans. Amer. Math. Soc., 77 (1954), 520-551.

4. M. Nakaoka, Cohomology theory of a complex with a transformation of prime period and its applications, J. Inst. Polytech. Osaka City Univ. Ser., A7 (1956), 51-102.

5. G. P. Porter, The homotopy groups of wedges of suspensions, Amer. J. Math., 88 (1966), 655-663.

6. V. P. Snaith, On cyclic maps, Proc. Camb. Phil. Soc., (to appear)

7. J. J. Ucci, On the symmetric cube of a sphere, Trans. Amer. Math. Soc., 151(1970), 527-549.

8. - On cyclic and iterated cyclic products of spheres, Osaka J. Math., 8(1971), $393-404$.

9. - Symmetric maps of least positive James number, Indiana University Math. J., 21 (1972), 709-714.

Received October 7, 1971.

Emmanuel College, Cambridge

AND

SYRACUSE UNIVERSITY 



\section{PACIFIC JOURNAL OF MATHEMATICS}

EDITORS

\author{
H. SAMELSON \\ Stanford University \\ Stanford, California 94305 \\ C. R. HOBBY \\ University of Washington \\ Seattle, Washington 98105
}

\author{
J. DUGUNDJI \\ Department of Mathematics \\ University of Southern California \\ Los Angeles, California 90007 \\ RICHARD ARENS \\ University of California \\ Los Angeles, California 90024
}

\section{ASSOCIATE EDITORS}

E. F. BECKENBACH

B. H. NeUManN

F. WOLF

K. YosHIDA

\section{SUPPORTING INSTITUTIONS}

UNIVERSITY OF BRITISH COLUMBIA

CALIFORNIA INSTITUTE OF TECHNOLOGY

UNIVERSITY OF CALIFORNIA

MONTANA STATE UNIVERSITY

UNIVERSITY OF NEVADA

NEW MEXICO STATE UNIVERSITY

OREGON STATE UNIVERSITY

UNIVERSITY OF OREGON

OSAKA UNIVERSITY
UNIVERSITY OF SOUTHERN CALIFORNIA

STANFORD UNIVERSITY

UNIVERSITY OF TOKYO

UNIVERSITY OF UTAH

WASHINGTON STATE UNIVERSITY UNIVERSITY OF WASHINGTON

AMERICAN MATHEMATICAL SOCIETY NAVAL WEAPONS CENTER 


\section{Pacific Journal of Mathematics}

\section{Vol. 45, No. $1 \quad$ September, 1973}

William George Bade, Complementation problems for the Baire classes .......... 1

Ian Douglas Brown, Representation of finitely generated nilpotent groups ........ 13

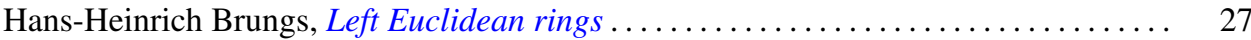

Victor P. Camillo and John Cozzens, A theorem on Noetherian hereditary rings ..... 35

James Cecil Cantrell, Codimension one embeddings of manifolds with locally flat

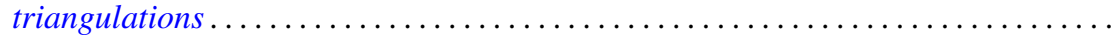

L. Carlitz, Enumeration of up-down permutations by number of rises . . . . . . . . . .

Thomas Ashland Chapman, Surgery and handle straightening in Hilbert cube

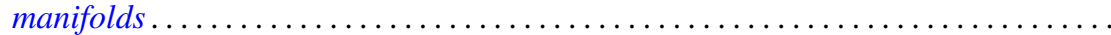

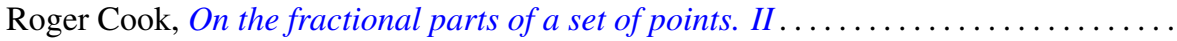

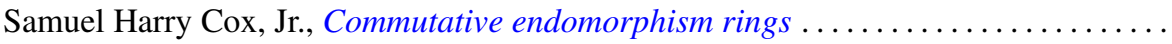

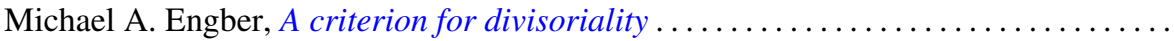

Carl Clifton Faith, When are proper cyclics injective . . . . . . . . . . . . . . 97

David Finkel, Local control and factorization of the focal subgroup . . . . . . . . . 113

Theodore William Gamelin and John Brady Garnett, Bounded approximation by

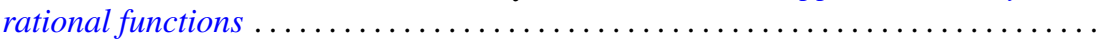

Kazimierz Goebel, On the minimal displacement of points under Lipschitzian

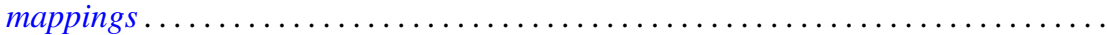

Frederick Paul Greenleaf and Martin Allen Moskowitz, Cyclic vectors for representations associated with positive definite measures: nonseparable

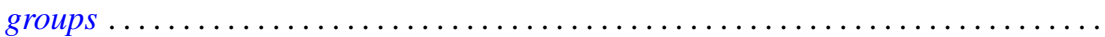

Thomas Guy Hallam and Nelson Onuchic, Asymptotic relations between perturbed linear systems of ordinary differential equations .

David Kent Harrison and Hoyt D. Warner, Infinite primes of fields and completions. .

James Michael Hornell, Divisorial complete intersections . ......

Jan W. Jaworowski, Equivariant extensions of maps ..............

John Jobe, Dendrites, dimension, and the inverse arc function .. .

Gerald William Johnson and David Lee Skoug, Feynman integrals of non-factorable

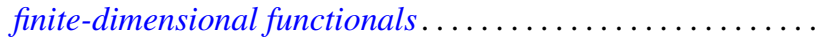

Dong S. Kim, A boundary for the algebras of bounded holomorphic functions ...... 269

Abel Klein, Renormalized products of the generalized free field and its derivatives ... 275

Joseph Michael Lambert, Simultaneous approximation and interpolation in $L_{1}$ and

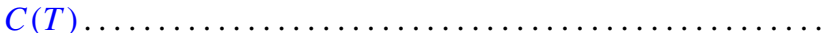

Kelly Denis McKennon, Multipliers of type $(p, p)$ and multipliers of the group

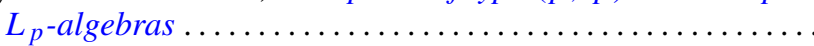

William Charles Nemitz and Thomas Paul Whaley, Varieties of implicative

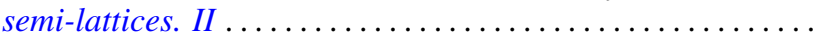

Donald Steven Passman, Some isolated subsets of infinite solvable

Norma Mary Piacun and Li Pi Su, Wallman compactifications on E-completely

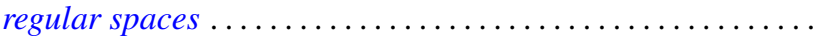

Jack Ray Porter and Charles I. Votaw, $S(\alpha)$ spaces and regular Hausdorff extensions....

Gary Sampson, Two-sided $L_{p}$ estimates of convolution transforms .

Ralph Edwin Showalter, Equations with operators forming a rig
Raymond Earl Smithson, Fixed points in partially ordered sets .

Victor Snaith and John James Ucci, Three remarks on symmetric products and

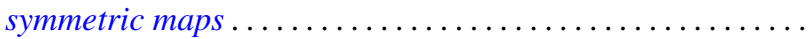

